\title{
Diversification-Driven Tabu Search for Unconstrained Binary Quadratic Problems
}

\author{
Fred Glover · Zhipeng Lü · Jin-Kao Hao
}

Received: date / Accepted: date

\begin{abstract}
This paper describes a Diversification-Driven Tabu Search $\left(\mathrm{D}^{2} \mathrm{TS}\right)$ algorithm for solving unconstrained binary quadratic problems. $\mathrm{D}^{2} \mathrm{TS}$ is distinguished by the introduction of a perturbation-based diversification strategy guided by long-term memory. The performance of the proposed algorithm is assessed on the largest instances from the ORLIB library (up to 2500 variables) as well as still larger instances from the literature (up to 7000 variables). The computational results show that $\mathrm{D}^{2} \mathrm{TS}$ is highly competitive in terms of both solution quality and computational efficiency relative to some of the best performing heuristics in the literature.
\end{abstract}

Keywords UBQP · Tabu Search · Diversification-Driven · Long-Term Memory

\section{Introduction}

The unconstrained binary quadratic programming problem may be written

$$
\begin{gathered}
\text { UBQP: } \text { Maximize } x_{o}=x Q x^{\prime} \\
x \text { binary }
\end{gathered}
$$

where $\mathrm{Q}$ is an $n$ by $n$ matrix of constants and $x$ is an $n$-vector of binary (zero-one) variables.

Fred Glover

OptTek Systems, Inc., 1919 Seventh Street Boulder, CO 80302, USA

E-mail: glover@opttek.com

Zhipeng Lü

LERIA, Université d'Angers, 2 Boulevard Lavoisier, 49045 Angers Cedex 01, France

E-mail: lu@info.univ-angers.fr, zhipeng.lui@gmail.com

Jin-Kao Hao

LERIA, Université d'Angers, 2 Boulevard Lavoisier, 49045 Angers Cedex 01, France

E-mail: hao@info.univ-angers.fr, http://www.info.univ-angers.fr/pub/hao/ 
The formulation UBQP is notable for its ability to represent a wide range of important problems, including those from social psychology (Harary (1953)), financial analysis (Laughunn (1970); McBride and Yormark (1980)), computer aided design (Krarup and Pruzan (1978)), traffic management (Gallo et al (1980); Witsgall (1975)), machine scheduling (Alidaee et al (1994)), cellular radio channel allocation (Chardaire and Sutter (1994)) and molecular conformation (Phillips and Rosen (1994)). Moreover, many combinatorial optimization problems pertaining to graphs such as determining maximum cliques, maximum cuts, maximum vertex packing, minimum coverings, maximum independent sets, maximum independent weighted sets are known to be capable of being formulated by the UBQP problem as documented in papers of Pardalos and Rodgers (1990), Pardalos and Xue (1994). A review of additional applications and formulations can be found in Kochenberger et al $(2004,2005)$, Alidaee et al (2008), Lewis et al (2008).

Given the interest of the UBQP, a large number of solution procedures have been reported in the literature. Some representative examples include local search based approaches such as Simulated Annealing (Alkhamis et al (1998); Beasley (1998); Katayama and Narihisa (2001)) and Tabu Search (Glover et al (1998); Beasley (1998); Palubeckis (2004, 2006)), populationbased approaches such as Evolutionary Algorithms (Lodi et al (1999); Merz and Freisleben (1999); Katayama et al (2000); Borgulya (2005)), Scatter Search (Amini et al (1999)) and Memetic Algorithms (Merz and Katayama (2004)).

Among these procedures, TS represents one of the most popular and successful approaches. One of the first adaptive memory TS algorithms for the UBQP (Glover et al (1998)), for instance, has since been used to solve applications arising in a wide variety of settings, as a demonstration of the value of the UBQP model and the ability to solve such applications successfully. More recently, Palubeckis (2004) has explored several multistart TS strategies and has achieved very good results on large problem instances. A sequel further improves these results by an Iterated Tabu Search algorithm (Palubeckis $(2006))$.

In the current paper, we introduce a new TS algorithm which employs a guided diversification strategy utilizing an information-based perturbation operator. We show that this Diversification-Driven Tabu Search $\left(D^{2} \mathrm{TS}\right)$ algorithm is highly effective in solving a large range of benchmark instances from the literature. For example, for the well-known UBQP instances containing up to 2500 variables (Beasley (1998)) that has been used in many published papers, $\mathrm{D}^{2} \mathrm{TS}$ attains the best known objective values in less than one minute. Moreover, for the set of 21 large instances containing 3000 to 7000 variables introduced in (Palubeckis $(2004,2006)$ ), our algorithm is able to match or even improve the best previous results. 


\section{Diversification-Driven TS $\left(\mathrm{D}^{2} \mathrm{TS}\right)$ for UBQP}

\subsection{Main idea of $\mathrm{D}^{2} \mathrm{TS}$}

$\mathrm{D}^{2} \mathrm{TS}$ repeatedly alternates between a simple version of Tabu Search that we denote by $\mathrm{TS}^{o}$ and a diversification phase founded on memory-based perturbation operator. Starting from an initial random solution, $\mathrm{D}^{2} \mathrm{TS}$ uses the $\mathrm{TS}^{o}$ procedure to reach a local optimum. Then, the perturbation operator is applied to displace the solution to a new region, whereupon a new round of $\mathrm{TS}^{\circ}$ is launched. To achieve a more effective diversification, the perturbation operator is guided by information from a special memory structure for obtaining improved results in this context. The next two sub-sections give a detailed explanation of the neighborhood and the tabu list management of the $\mathrm{TS}^{\circ}$ procedure, as well as the memory-based perturbation operator.

\subsection{Neighborhood and tabu list}

\subsubsection{Neighborhood using 1-Flip moves}

Our $\mathrm{TS}^{\circ}$ procedure uses a neighborhood defined by the well-known 1-flip move, which consists of changing (flipping) the value of a single variable $x_{i}$ to its complementary value $1-x_{i}$. It is clear that the size of this neighborhood is bounded by $O(n)$, i.e., at most $n$ moves are required to go from any solution to any other solution.

For large problem instances, it is imperative to be able to rapidly determine the effect of a move on the objective function $x_{o}$. For this purpose, we employ a fast incremental evaluation technique first introduced by Glover et al (1998) and enhanced by Glover and Hao (2009a) to exploit an improved representation and to take advantage of sparse data - a characteristic of many real world problems. The procedure maintains a data structure that stores the move value (change in $x_{o}$ ) for each possible move, and employs a streamlined calculation for updating this data structure after each iteration.

The key elements of this procedure may be summarized as follows. Let $N=\{1, \ldots, n\}$ denote the index set for components of the $x$ vector. We preprocess the matrix $Q$ to put it in lower triangular form by redefining (if necessary) $q_{i j}=q_{i j}+q_{j i}$ for $i>j$, which is implicitly accompanied by setting $q_{j i}=0$ (though these 0 entries above the main diagonal are not stored or accessed). Let $\Delta x_{i}$ be the move value of flipping the variable $x_{i}$, and let $q_{(i, j)}$ be a shorthand for denoting $q_{i j}$ if $i>j$ and $q_{j i}$ if $j>i$. Then each can be calculated in linear time using the formula:

$$
\Delta x_{i}=\left(1-2 x_{i}\right)\left(q_{i i}+\sum_{j \in N, j \neq i, x_{j}=1} q_{(i, j)}\right)
$$


Significantly, it is possible to update the move values upon flipping a variable $x_{i}$ by performing the following abbreviated calculation, using the convention that $x_{i}$ represents $x_{i}$ 's value before being flipped.

1. $\Delta x_{i}=-\Delta x_{i}$

2. For each $j \in N-\{i\}$,

$\Delta x_{j}=\Delta x_{j}+\sigma_{i, j} \cdot q_{(i, j)}$

where $\sigma_{i, j}=1$ if $x_{i}=x_{j}$ and $\sigma_{i, j}=-1$ otherwise.

These updates can be implemented highly efficiently in the presence of sparse data using the procedures in Glover and Hao (2009a).

\subsubsection{Tabu list management}

TS typically incorporates a tabu list as a "recency-based" memory structure to assure that solutions visited within a certain span of iterations, called the tabu tenure, will not be revisited (Glover and Laguna (1997)). The approach is designed to introduce vigor into the search by also forbidding moves leading to related solutions that share certain attributes (values of variables) in common with the visited solutions. In our present implementation we use a simple tabu list consisting of an n-vector TabuTenure $(i), i \in N$. When the variable $x_{i}$ is flipped, we have elected to set

$$
\text { TabuTenure }(i)=c+\operatorname{rand}(10)
$$

where $c$ is a constant and rand(10) denotes a randomly generated number from 1 to 10 . The constant $c$ is determined according to the size of the problem instance and is experimentally fixed at $n / 100$ in our implementation.

This tabu list assignment is used to prevent $x_{i}$ from being flipped until a number of TabuTenure $(i)$ iterations have elapsed. (To facilitate implementation, TabuTenure $(i)$ is customarily increased by the value of the current iteration at the time when the assignment (1) is made, and this modified value is checked against subsequent values of the iteration counter.) The $\mathrm{TS}^{\circ}$ algorithm then restricts consideration to variables not forbidden by the tabu list, and selects a variable to flip that produces the largest $\Delta x_{i}$ value (thus improving $x_{o}$ if this value is positive). Accompanying this rule, a simple aspiration criterion is applied that permits a move to be selected in spite of being tabu if it leads to a solution better than the current best solution.

This rudimentary TS process stops when the best solution cannot be improved within a given number $\alpha$ of moves that we call the improvement cutoff.

\subsection{Diversification Phase}

In order to enhance the diversification capability of the preceding $\mathrm{TS}^{\circ}$ algorithm, we introduce a strategy which relies on a memory-based perturbation operator composed of three parts: a flip frequency memory (FlipFreq), an elite 
solution memory (EliteSol) and an elite value frequency memory (EliteFreq). These memory structures are used jointly by the perturbation operator (see Section 2.3.2).

\subsubsection{Memory management}

Our tabu search procedure uses the vector FlipFreq $(i), i \in N$ to record the number of times the variable $x_{i}$ has been flipped. This information is used to guide the design of the scoring function of the perturbation operator (see Section 2.3.2).

EliteSol stores a set of elite locally optimal solutions found by $\mathrm{TS}^{o}$ using a design commonly employed to construct reference sets in scatter search methods. We represent this memory as a list EliteSol $=\left[S_{1}, \ldots, S_{R}\right]$, where $R$ is a maximum allowed dimension of EliteSol and $S_{i}$ represents the $i$ th local optimum recorded in this memory. In our implementation, $R$ was set to be 8 for all the problems we have tested in this paper. The first solution inserted on EliteSol is the best solution obtained by the first phase of the $\mathrm{TS}^{\circ}$ procedure. After that, new local optima obtained by successive runs of the $\mathrm{TS}^{\circ}$ procedure are added to the list provided they do not already exist in the memory, continuing until $R$ different solutions are stored. From this point on, each time a new local optimum is found that has an $x_{o}$ value superior to that of the worst local optimum on EliteSol, the new solution replaces this worst element. The resulting pool of high quality solutions provides a source of candidates for applying the perturbation operator.

Finally, the vector EliteFreq(i), $i \in N$ records the total number of times variable $x_{i}$ is assigned value 1 in the elite solutions currently stored in EliteSol. This memory is used to penalize the use of flips during the perturbation phase for variables that have more consistently received the same value in the elite solutions, thus constituting a form of intensification process that favors retaining the value assignments that occur more often in the best solutions found to date. See Section 2.3.2 for more details.

\subsubsection{Memory-based perturbation operator}

From a general perspective, the perturbation component of the diversification phase has two aims: to jump out of local optima and to lead the search procedure to a new promising region. In our case, the perturbation step first randomly selects an elite solution from the list EliteSol and then applies a perturbation operator to the selected solution. Contrary to a conventional random perturbation strategy, our perturbation operator uses the so-called critical element-guided perturbation strategy (Lü and Hao (2009)), which is composed of three steps: 1) Scoring: assign a score to each variable; 2) Selection: choose a certain number of highly-scored variables (critical elements); 3 ) Perturbing: perturb the solution using the chosen critical elements.

The scoring function ranks each variable by taking into account its flip frequency $($ FlipFreq $(i)$ and its elite value frequency $($ EliteFreq $(i))$. Let $r$ 
$(0 \leq r \leq R)$ be the current number of solutions recorded in EliteSol, our scoring function takes the following form:

$$
\operatorname{Score}\left(x_{i}\right)=\frac{\operatorname{EliteFreq}(i)(r-\operatorname{EliteFreq}(i))}{r^{2}}+\beta\left(1-\frac{\text { FlipFreq }(i)}{\text { max_Freq }}\right)
$$

where $\beta$ is a constant and max_Freq is the largest of the FlipFreq $(i)$ values, i.e., $\max _{-}$Freq $=\max _{i=1, \ldots, N}\left\{\right.$ FlipFreq $_{(i)\}}$. In this paper, we set $\beta=0.3$ for all our experiments.

The first part of the score function is based on the supposition that a variable $x_{i}$ whose EliteFreq $(i)$ value equals an extreme value 0 or $r$ should be given little opportunity to be flipped since it always receives the same value in the elite solutions in the memory. On the other hand, a variable $x_{i}$ whose EliteFreq $(i)$ value equals $r / 2$ should have complete freedom to change its value. The basic idea behind the second part of the score function (3) is to give a high flip probability to a variable that is seldom flipped. Our supposition is that changing the value of such a variable can help the search to jump out of local optima.

For the selection step, we first sort all the variables in non-increasing order according to their scores and then probabilistically select $\gamma$ different variables to be randomly assigned a value 0 or 1 ( $\gamma$ is called the perturbation strength). This selection procedure is implemented in an adaptive way, i.e., the higher the score a variable has, the greater the probability it will be chosen. The $j$ th highly-scored variable is selected to be flipped according to the probability:

$$
P_{j}=\frac{j^{-\lambda}}{\sum_{i=1}^{n} i^{-\lambda}}
$$

where $\lambda$ is a positive number. Note that this selection procedure is problem independent.

Finally for the perturbation step, we just flip the values of the selected critical variables. This perturbed solution is then used to initiate a new round of our tabu search procedure by once again launching $\mathrm{TS}^{o}$. Computational experiments presented in Section 3 confirm the value of this special form of perturbation as a diversification strategy for solving large scale UBQP instances.

\subsection{D² TS Algorithm Description}

Our $\mathrm{D}^{2} \mathrm{TS}$ algorithm is summarized in Algorithm 1.

Some brief comments are appropriate. At the beginning of the search, the EliteSol list is empty with $r=0$. The first loop from lines 5 to 14 fills the list one element at a time until the number of elements in EliteSol reaches its given limit $R$. The EliteFreq vector is also updated at each iteration. The loop in lines 15 to 24 repeatedly updates EliteSol and the EliteFreq vector until a specified stop condition is met. In this loop, if a new locally optimal 


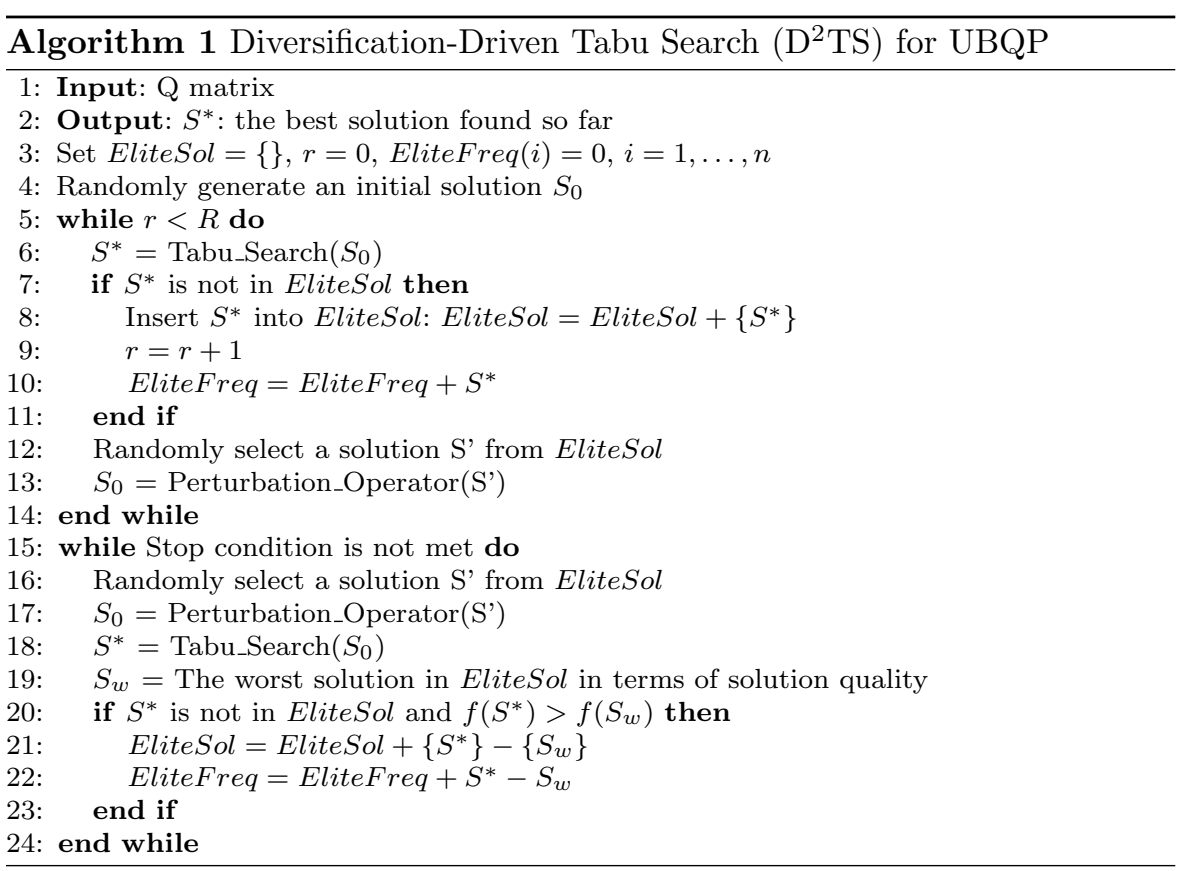

solution $S^{*}$ is better than the worst solution $S_{w}$ in EliteSol and if $S^{*}$ does not exist in EliteSol, then $S^{*}$ replaces $S_{w}$ on this list.

\section{Computational Results}

To assess the efficiency of our proposed $\mathrm{D}^{2} \mathrm{TS}$ algorithm, we carry out experiments on 31 medium and large instances in the literature and compare $\mathrm{D}^{2} \mathrm{TS}$ with five best performing algorithms. At the end of this section, we provide an experimental analysis demonstrating the importance of the memory-based perturbation operator described in Section 2.3.2.

\subsection{Experimental protocol}

Our algorithm is programmed in $\mathrm{C}$ and compiled using GNU GCC on a PC running Windows XP with Pentium 2.66GHz CPU and 512M RAM. All computational results were obtained without special tuning of the parameters, i.e., all the parameters used in our algorithm are fixed (constant) or dynamically and automatically tuned during the problem solving for all instances considered. It is possible that better solutions would be found by using a set of instance-dependent parameters. However, our aim is to design a robust solver that is able to solve a large panel of instances efficiently. Table 1 gives the descriptions and settings of the parameters used in our $\mathrm{D}^{2} \mathrm{TS}$ algorithm. These 
Table 1 Settings of important parameters

\begin{tabular}{llll}
\hline Parameters & Section & Description & Values \\
\hline$c$ & 2.2 .1 & tabu tenure constant & $n / 100$ \\
$\alpha$ & 2.2 .2 & improvement cutoff of TS & $20 n$ \\
$R$ & 2.3 .1 & maximum size of the memory EliteSol & 8 \\
$\beta$ & 2.3 .2 & frequency-related weight in perturbation scoring & 0.3 \\
$\lambda$ & 2.3 .2 & perturbation selection importance factor & 1.2 \\
$\gamma$ & 2.3 .2 & perturbation strength & $n / 4$ \\
\hline
\end{tabular}

parameters are tuned in two steps. We calibrate first the two parameters of $\mathrm{TS}^{0}$ (tabu tenure constant and improvement cutoff), followed by fixing the perturbation operator. Based on preliminary testing, we observed that the following parameter settings give satisfying results: $c \in[n / 200, n / 80], \alpha \in[10 n, 50 n]$, $R \in[6,15], \beta \in[0.2,0.5], \lambda \in[1.1,1.4]$ and $\gamma \in[n / 5, n / 3]$. The calibrated parameter values are kept constant for all the experiments. It is possible that better solutions would be found by using a set of instance-dependent parameters.

\subsection{Test instances}

Two sets of test problems are considered in the experiments, in total constituting 31 instances. The first set of benchmarks is composed of 10 largest instances of size $n=2500$ introduced in (Beasley (1998)) and available in the ORLIB (Beasley (1996)). They all have a density of 0.1 and are named by b2500.1,..,b2500.10. These instances are used in the literature by many authors, see for instance (Beasley (1998); Katayama and Narihisa (2001); Merz and Freisleben (2002); Merz and Katayama (2004); Palubeckis (2004, 2006)). The second set of benchmarks consists of a set of 21 randomly generated large problem instances named p3000.1,..,p7000.3 with sizes ranging from $n=3000$ to 7000 and with densities from 0.5 to 1.0 (Palubeckis $(2004,2006)$ ). Nonzero entries of $Q$ are drawn uniformly from the interval [-100, 100]. The sources of the generator and input files to replicate these problem instances can be found at: http://www.soften.ktu.lt/ gintaras/ubqop_its.html. Experiments reported in (Palubeckis $(2004,2006))$ showed that these large instances are particularly challenging for UBQP algorithms.

The small test instances from the ORLIB whose sizes range from $n=500$ to 1000 and the similarly small instances from (Glover et al (1998)) are not considered here, since they are solved very easily within 30 seconds by our algorithm and are also solved relatively easily by most recent heuristics.

\subsection{Computational results on ORLIB instances}

Our first experiment aims to evaluate the $\mathrm{D}^{2} \mathrm{TS}$ algorithm on the 10 ORLIB instances with 2500 variables. The results of this experiment are summarized in Tables 2 to 4 . 
Table 2 Results of $\mathrm{D}^{2} \mathrm{TS}$ algorithm on the Beasley instances from ORLIB

\begin{tabular}{c|ccccccc}
\hline instance & dens & $f_{\text {prev }}$ & \multicolumn{5}{c}{$\mathrm{D}^{2} \mathrm{TS}$ Algorithm } \\
\cline { 3 - 7 } & & & $f_{\text {best }}$ & $f_{\text {best }}-f_{\text {prev }}$ & $f_{\text {aver }}$ & success & seconds \\
\hline b2500.1 & 0.1 & 1515944 & 1515944 & 0 & 1515944 & $25 / 25$ & 6 \\
b2500.2 & 0.1 & 1471392 & 1471392 & 0 & 1471392 & $25 / 25$ & 38 \\
b2500.3 & 0.1 & 1414192 & 1414192 & 0 & 1414192 & $25 / 25$ & 35 \\
b2500.4 & 0.1 & 1507701 & 1507701 & 0 & 1507701 & $25 / 25$ & 4 \\
b2500.5 & 0.1 & 1491816 & 1491816 & 0 & 1491816 & $25 / 25$ & 5 \\
b2500.6 & 0.1 & 1469162 & 1469162 & 0 & 1469162 & $25 / 25$ & 10 \\
b2500.7 & 0.1 & 1479040 & 1479040 & 0 & 1479040 & $25 / 25$ & 20 \\
b2500.8 & 0.1 & 1484199 & 1484199 & 0 & 1484199 & $25 / 25$ & 12 \\
b2500.9 & 0.1 & 1482413 & 1482413 & 0 & 1482413 & $25 / 25$ & 6 \\
b2500.10 & 0.1 & 1483355 & 1483355 & 0 & 1483355 & $25 / 25$ & 7 \\
\hline
\end{tabular}

Table 2 shows the computational statistics of our $\mathrm{D}^{2} \mathrm{TS}$ algorithm. Columns 2 and 3 respectively give the density (dens) and the previous best known results $\left(f_{\text {prev }}\right)$. Columns 4 to 8 give our results: the best objective value $\left(f_{\text {best }}\right)$, the difference between our best values with the previous best known values $\left(f_{\text {best }}-f_{\text {prev }}\right)$, the average objective value $\left(f_{\text {aver }}\right)$, the success rate (success) and the average CPU time (seconds) for reaching the best result $\left(f_{\text {best }}\right)$. Table 2 discloses that our $\mathrm{D}^{2} \mathrm{TS}$ algorithm can stably reach all the previous best known results within 40 seconds on our computer, demonstrating the high efficiency of our method.

Table 3 shows the average results of our $\mathrm{D}^{2} \mathrm{TS}$ algorithm compared with the five leading algorithms in the literature, respectively named ITS (Palubeckis (2006)), MST1 (Palubeckis (2004)), MST2 (Palubeckis (2004)), SA (Katayama and Narihisa (2001)) and MA (Merz and Katayama (2004)). The results of these five algorithms are extracted from (Palubeckis (2006)) and have been obtained by Palubeckis by applying each under the same experimental conditions, which we likewise employ for evaluating our algorithm. These five algorithms were run 25 times for each problem instance with a time limit of 600 seconds on a Pentium III 800 PC. Since our computer is about 3 times faster than that used by Palubeckis (2006), we limit the running CPU time of $\mathrm{D}^{2} \mathrm{TS}$ to 200 seconds $^{1}$. The overall results, averaged over 10 instances, are presented in the last row. From Table 3, one observes that our $\mathrm{D}^{2} \mathrm{TS}$ algorithm obtains the previous best known results more stably than these alternative heuristics that are reported to be the most effective in the literature.

Table 4 compares the average time (in seconds) needed by each of the compared algorithms to hit the best objective value in the run. We have converted our CPU time reported in Table 2 by multiplying it by 3 to compensate for the fact that our computer is about 3 times faster. A corresponding conversion also applies to Tables 7 and 8 . From Table 4 , we observe that $\mathrm{D}^{2} \mathrm{TS}$ can easily obtain the previous best known solutions within 120 seconds (con-

1 We tested a benchmark program on our computer and a Pentium III 800 PC with 512M memory and found that the exact speed ratio of these two computers is 2.92. This benchmark program is used by the second International Timetabling Competition and available at: http://www.cs.qub.ac.uk/itc2007/benchmarking/benchmark_machine.zip. 
Table 3 Average performance of $\mathrm{D}^{2} \mathrm{TS}$ and other algorithms on the Beasley problems

\begin{tabular}{c|ccccccc}
\hline instance & $f_{\text {prev }}$ & \multicolumn{6}{c}{ Solution difference (i.e., average heuristic value - $f_{\text {prev }}$ ) } \\
\cline { 2 - 7 } & & $\mathrm{D}^{2}$ TS & ITS & MST1 & MST2 & SA & MA \\
\hline b2500.1 & 1515944 & 0 & 0 & 0 & 0 & -4 & -13 \\
b2500.2 & 1471392 & 0 & -9 & -133 & 0 & -433 & -645 \\
b2500.3 & 1414192 & 0 & -11 & 0 & -11 & -117 & -173 \\
b2500.4 & 1507701 & 0 & 0 & 0 & 0 & 0 & 0 \\
b2500.5 & 1491816 & 0 & 0 & 0 & 0 & -6 & -55 \\
b2500.6 & 1469162 & 0 & 0 & -1 & 0 & -58 & -190 \\
b2500.7 & 1479040 & 0 & 0 & -4 & 0 & -208 & -416 \\
b2500.8 & 1484199 & 0 & 0 & 0 & 0 & -35 & -3 \\
b2500.9 & 1482413 & 0 & 0 & 0 & 0 & -33 & -321 \\
b2500.10 & 1483355 & 0 & 0 & -8 & 0 & -493 & -446 \\
\hline Average & & 0 & -2 & -15 & -1 & -139 & -226 \\
\hline
\end{tabular}

Table 4 Average time performance of $\mathrm{D}^{2} \mathrm{TS}$ and other algorithms on the Beasley problems: average time to the best solution in the run (in seconds)

\begin{tabular}{c|cccccc}
\hline instance & $\mathrm{D}^{2} \mathrm{TS}$ & ITS & MST1 & MST2 & SA & MA \\
\hline b2500.1 & 18 & 18 & 14 & 13 & 225 & 461 \\
b2500.2 & 114 & 205 & 281 & 158 & 334 & 430 \\
b2500.3 & 105 & 196 & 91 & 134 & 319 & 422 \\
b2500.4 & 12 & 6 & 8 & 9 & 120 & 293 \\
b2500.5 & 15 & 12 & 7 & 11 & 305 & 469 \\
b2500.6 & 30 & 22 & 48 & 23 & 283 & 452 \\
b2500.7 & 60 & 75 & 168 & 99 & 387 & 478 \\
b2500.8 & 36 & 46 & 26 & 47 & 293 & 359 \\
b2500.9 & 18 & 54 & 77 & 71 & 340 & 450 \\
b2500.10 & 21 & 104 & 161 & 138 & 351 & 477 \\
\hline Average & 42 & 74 & 88 & 70 & 296 & 429
\end{tabular}

verted time). From Tables 2 to 4 , we conclude that $\mathrm{D}^{2} \mathrm{TS}$ is quite competitive compared with these reference algorithms in terms of both solution quality and computational efficiency. However, from the results presented above, it is impossible to conclude that any given algorithm dominates the others since the problem instances in this set are not sufficiently difficult to solve. More significant differences are observed when larger and harder instances are used, as we show next.

\subsection{Computational results on larger instances}

In the second experiment we tested our $\mathrm{D}^{2} \mathrm{TS}$ algorithm on the second set of 21 randomly generated instances ${ }^{2}$. These instances of larger size and higher density are more difficult for the search algorithms. Tables 5 reports the computational results obtained by $\mathrm{D}^{2} \mathrm{TS}$ for solving these instances, following the same format as Table 2. The stop condition is set to be the same as in (Palubeckis (2006)), i.e., the cutoff time for a run is 15, 30, 60, 90 and 150 minutes on a Pentium III 800 PC for an instance with 3000, 4000, 5000, 6000 and 7000

2 Our best results are available at: http://www.info.univ-angers.fr/pub/hao/UBQP.html 
Table 5 Results of our $\mathrm{D}^{2} \mathrm{TS}$ algorithm on 21 large random problem instances with size from $\mathrm{n}=3000$ to $\mathrm{n}=7000$

\begin{tabular}{l|ccccccc}
\hline instance & dens & $f_{\text {prev }}$ & \multicolumn{5}{c}{$\mathrm{D}^{2} \mathrm{TS}$ Algorithm } \\
\cline { 3 - 7 } & & & $f_{\text {best }}$ & $f_{\text {best }}-f_{\text {prev }}$ & $f_{\text {aver }}$ & success & seconds \\
\hline p3000.1 & 0.5 & 3931583 & 3931583 & 0 & 3931583 & $20 / 20$ & 70 \\
p3000.2 & 0.8 & 5193073 & 5193073 & 0 & 5193073 & $20 / 20$ & 82 \\
p3000.3 & 0.8 & 5111533 & 5111533 & 0 & 5111533 & $20 / 20$ & 79 \\
p3000.4 & 1.0 & 5761822 & 5761822 & 0 & 5761822 & $20 / 20$ & 111 \\
p3000.5 & 1.0 & 5675625 & 5675625 & 0 & 5675625 & $20 / 20$ & 159 \\
p4000.1 & 0.5 & 6181830 & 6181830 & 0 & 6181830 & $20 / 20$ & 91 \\
p4000.2 & 0.8 & 7801355 & 7801355 & 0 & 7801355 & $20 / 20$ & 252 \\
p4000.3 & 0.8 & 7741685 & 7741685 & 0 & 7741685 & $20 / 20$ & 178 \\
p4000.4 & 1.0 & 8711822 & 8711822 & 0 & 8711822 & $20 / 20$ & 223 \\
p4000.5 & 1.0 & 8908979 & 8908979 & 0 & 8908979 & $20 / 20$ & 702 \\
p5000.1 & 0.5 & 8559355 & 8559355 & 0 & 8559024 & $6 / 10$ & 2855 \\
p5000.2 & 0.8 & 10836019 & 10836019 & 0 & 10823486 & $8 / 10$ & 1155 \\
p5000.3 & 0.8 & 10489137 & 10489137 & 0 & 10476261 & $7 / 10$ & 1326 \\
p5000.4 & 1.0 & 12251874 & 12252318 & 444 & 12250356 & $4 / 10$ & 838 \\
p5000.5 & 1.0 & 12731803 & 12731803 & 0 & 12731564 & $9 / 10$ & 623 \\
p6000.1 & 0.5 & 11384976 & 11384976 & 0 & 11384976 & $10 / 10$ & 509 \\
p6000.2 & 0.8 & 14333855 & 14333855 & 0 & 1432569 & $5 / 10$ & 1543 \\
p6000.3 & 1.0 & 16132915 & 16132915 & 0 & 1613128 & $4 / 10$ & 2088 \\
p7000.1 & 0.5 & 14478336 & 14478676 & 340 & 1446538 & $4 / 10$ & 1217 \\
p7000.2 & 0.8 & 18248297 & 18249844 & 1547 & 18241236 & $7 / 10$ & 849 \\
p7000.3 & 1.0 & 20446407 & 20446407 & 0 & 2043856 & $3 / 10$ & 3520 \\
\hline
\end{tabular}

variables, respectively. (The time limit on our computer is set to be $1 / 3$ of these values.) Column 5 shows that under this stop condition our $\mathrm{D}^{2} \mathrm{TS}$ algorithm matches the previous best known results for 18 instances and improves the previous best known results for 3 instances, named p5000.4, p7000.1 and p7000.2.

In order to further compare our $\mathrm{D}^{2} \mathrm{TS}$ algorithm with the best competing algorithms, we again refer to the algorithms used in Table 3 (ITS, MST1, MST2, SA and MA). As before, the results of the reference algorithms are directly extracted from (Palubeckis (2006)). Table 6 displays the solution difference between the best solutions obtained by these 6 algorithms with the best known results overall. The averaged results over the 21 instances are presented in the last row. From Table 6 it may be observed that our $\mathrm{D}^{2} \mathrm{TS}$ algorithm outperforms these five reference algorithms in terms of the quality of the best solution obtained. Notably, our $\mathrm{D}^{2} \mathrm{TS}$ algorithm finds better solutions than any of these five references algorithms for at least 4 instances (roughly $20 \%$ of the problems).

In (Palubeckis (2006)), the author identifies ITS as the top performing algorithm among the considered algorithms and reports computational results on five instances with 5000, 6000 and 7000 variables with longer runs of ITS. The time limit was then set at 5, 8 and 10 hours, respectively. For these five instances, the ITS algorithm improved its previous best results reported in Table 6 , as shown in Table 8 .

To check whether our $\mathrm{D}^{2} \mathrm{TS}$ algorithm is also able to improve its previous best results by allowing greater computational time, we re-ran $\mathrm{D}^{2} \mathrm{TS}$ on these 
Table 6 Best results comparison between $\mathrm{D}^{2} \mathrm{TS}$ and other state-of-the-art algorithms for larger problem instances

\begin{tabular}{l|cccccccc}
\hline instance & dens & $f_{\text {prev }}$ & \multicolumn{6}{c}{ solution difference (i.e., heuristic solution value - $f_{\text {prev }}$ ) } \\
\cline { 3 - 8 } & & & $\mathrm{D}^{2}$ TS & ITS & MST1 & MST2 & SA & MA \\
\hline p3000.1 & 0.5 & 3931583 & 0 & 0 & 0 & 0 & 0 & -3950 \\
p3000.2 & 0.8 & 5193073 & 0 & 0 & 0 & 0 & 0 & -342 \\
p3000.3 & 0.8 & 5111533 & 0 & 0 & -357 & 0 & 0 & 0 \\
p3000.4 & 1.0 & 5761822 & 0 & 0 & 0 & 0 & 0 & -1097 \\
p3000.5 & 1.0 & 5675625 & 0 & 0 & -478 & 0 & 0 & -478 \\
p4000.1 & 0.5 & 6181830 & 0 & 0 & 0 & 0 & 0 & -2390 \\
p4000.2 & 0.8 & 7801355 & 0 & 0 & -1686 & 0 & -504 & -6564 \\
p4000.3 & 0.8 & 7741685 & 0 & 0 & -54 & 0 & 0 & -5760 \\
p4000.4 & 1.0 & 8711822 & 0 & 0 & 0 & 0 & 0 & -2359 \\
p4000.5 & 1.0 & 8908979 & 0 & 0 & 0 & 0 & 0 & -9028 \\
p5000.1 & 0.5 & 8559355 & 0 & -375 & -2691 & 0 & -1107 & -4647 \\
p5000.2 & 0.8 & 10836019 & 0 & 0 & 0 & -582 & -582 & -7519 \\
p5000.3 & 0.8 & 10489137 & 0 & 0 & -3277 & 0 & -354 & -11552 \\
p5000.4 & 1.0 & 12251874 & 444 & -490 & -3341 & -1199 & 0 & -15955 \\
p5000.5 & 1.0 & 12731803 & 0 & 0 & -5150 & 0 & -1025 & -6644 \\
p6000.1 & 0.5 & 11384976 & 0 & 0 & -3198 & 0 & -430 & -9046 \\
p6000.2 & 0.8 & 14333855 & 0 & -88 & -10001 & 0 & -675 & -21732 \\
p6000.3 & 1.0 & 16132915 & 0 & -2729 & -11658 & 0 & 0 & -13400 \\
p7000.1 & 0.5 & 14478336 & 340 & 0 & -6778 & -1267 & -2239 & -13365 \\
p7000.2 & 0.8 & 18248297 & 1547 & 0 & -7251 & -679 & -3901 & -18898 \\
p7000.3 & 1.0 & 20446407 & 0 & 0 & -17652 & 0 & -2264 & -14684 \\
\hline Average & & & 126 & -175 & -3503 & -177 & -623 & -8067 \\
\hline
\end{tabular}

five instances using the same timing conditions used by ITS. The new results appear in Table 8. $\mathrm{D}^{2} \mathrm{TS}$ likewise improves its results for two out of five instances, matching the results of ITS for four instances and finding a better solution than ITS for the remaining instance (p5000.1).

\subsection{Influence of the Adaptive Memory Mechanism}

We turn our attention now to analyzing one of the most important components of the proposed $\mathrm{D}^{2} \mathrm{TS}$ algorithm, the memory-based perturbation operator described in Section 2.3.2. This strategy involves randomly and adaptively selecting and flipping a given number of highly-scored variables. We believe that constraining the choices to the critical variables is essential for our $\mathrm{D}^{2} \mathrm{TS}$ algorithm. In order to be sure this mechanism is meaningful, we carried out additional experiments to examine the influence of the proposed memory-based perturbation operator (denoted by $M B P$ ).

For this purpose, we compare $M B P$ with a pure random perturbation operator (denoted by $P R P$ ) where the variables to be flipped are totally uniformly selected without using any memory information. In order to observe the difference between these two perturbation strategies, we disable the memory-based perturbation within the $\mathrm{D}^{2} \mathrm{TS}$ algorithm and replace it by the random one while keeping other components unchanged. The algorithm stops after performing 100 perturbation operations. All other parameters are set as described 
Table 7 Time comparison between $\mathrm{D}^{2} \mathrm{TS}$ and other algorithms on larger problems: average time to the best solution in the run (in seconds)

\begin{tabular}{l|cccccc}
\hline instance & D $^{2}$ TS & ITS & MST1 & MST2 & SA & MA \\
\hline p3000.1 & 209 & 228 & 396 & 106 & 251 & 726 \\
p3000.2 & 245 & 212 & 395 & 97 & 337 & 809 \\
p3000.3 & 237 & 327 & 464 & 271 & 517 & 590 \\
p3000.4 & 334 & 519 & 480 & 559 & 336 & 722 \\
p3000.5 & 476 & 462 & 436 & 255 & 327 & 638 \\
p4000.1 & 274 & 215 & 776 & 436 & 842 & 1515 \\
p4000.2 & 756 & 1070 & 785 & 1082 & 1680 & 1063 \\
p4000.3 & 534 & 730 & 1011 & 359 & 1094 & 1106 \\
p4000.4 & 678 & 845 & 656 & 624 & 1002 & 1373 \\
p4000.5 & 2106 & 797 & 862 & 700 & 1279 & 1287 \\
p5000.1 & 3368 & 1520 & 2260 & 1621 & 1816 & 3000 \\
p5000.2 & 3465 & 1264 & 1984 & 1946 & 2072 & 2562 \\
p5000.3 & 3278 & 2015 & 1410 & 2365 & 2836 & 2925 \\
p5000.4 & 2513 & 1787 & 2005 & 2805 & 3178 & 2075 \\
p5000.5 & 1869 & 1652 & 1922 & 2156 & 3171 & 3095 \\
p6000.1 & 1527 & 2935 & 2860 & 3112 & 1844 & 4009 \\
p6000.2 & 4628 & 2517 & 3119 & 2661 & 3256 & 3688 \\
p6000.3 & 5264 & 2871 & 3217 & 3655 & 4422 & 4364 \\
p7000.1 & 4649 & 5313 & 4954 & 4348 & 5806 & 7942 \\
p7000.2 & 2547 & 3039 & 4484 & 5165 & 5215 & 5525 \\
p7000.3 & 8436 & 4339 & 2801 & 6342 & 6417 & 8197 \\
\hline Average & 2257 & 1650 & 1775 & 1936 & 2271 & 2724 \\
\hline
\end{tabular}

Table 8 Results of longer runs of ITS and $\mathrm{D}^{2} \mathrm{TS}$

\begin{tabular}{c|cccc}
\hline instance & $\mathrm{D}^{2} \mathrm{TS}$ & time $(\mathrm{s})$ & ITS & time(s) \\
\hline p5000.1 & $\mathbf{8 5 5 9 6 8 0}$ & 4531 & 8559355 & 3457 \\
p5000.4 & 12252318 & 1698 & 12252318 & 12605 \\
p6000.3 & 16132915 & 3125 & 16132915 & 9830 \\
p7000.1 & 14478676 & 6214 & 14478676 & 30198 \\
p7000.2 & 18249948 & 8423 & 18249948 & 1877 \\
\hline
\end{tabular}

in Subsection 3.1. For the purpose of illustration, we choose two large instances with 5000 variables (p5000.1 and p5000.4 with density equal to 0.5 and 1.0, respectively) as our test bed.

Figure 1 shows the running profiles of the two perturbation strategies. Each point represents the best solution cost (averages over 10 independent runs) found at the moment of each perturbation. It is easy to observe that on both instances the $M B P$ strategy obtains better results than the PRP strategy, especially when the perturbation iterations become large. We found the same results to occur in other instances.

\section{Discussion and Conclusion}

Our Diversification-Driven Tabu Search $\left(\mathrm{D}^{2} \mathrm{TS}\right)$ algorithm for solving unconstrained binary quadratic problems alternates between a rudimentary tabu search procedure $\left(\mathrm{TS}^{\circ}\right)$ and a memory-based perturbation strategy specially 


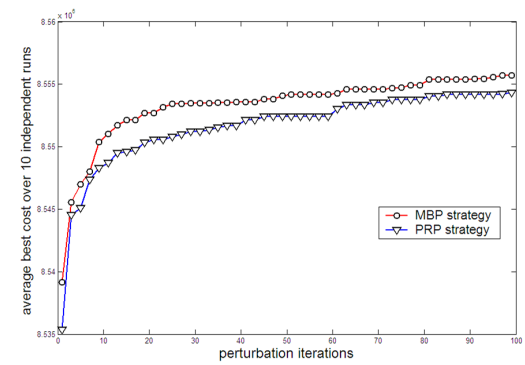

(a) Instance $\mathrm{p} 5000$.

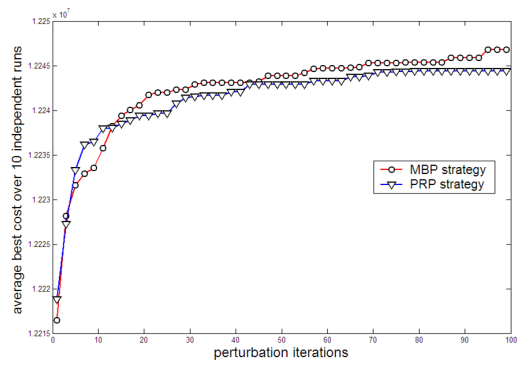

(b) Instance $\mathrm{p} 5000.4$

Fig. 1 Comparison between the memory-based perturbation operator with the pure random perturbation operator.

designed to achieve diversification. In spite of being quite simple in comparison with most top performing algorithms, $\mathrm{D}^{2} \mathrm{TS}$ proves to be highly effective in finding good solutions for two sets of 31 benchmark instances of medium and large sizes, containing from 2500 to 7000 variables. Compared with the five state-of-the-art algorithms from the literature, $\mathrm{D}^{2} \mathrm{TS}$ is able to find all the previous best known solutions (which none of the previous methods succeeded in doing) and obtains a new best, previously unknown, solution for one instance of 5000 variables.

There are several directions to extend this work. One immediate possibility is to examine other neighborhoods. $\mathrm{D}^{2} \mathrm{TS}$ and most existing algorithms are based on the simple 1-flip neighborhood. Richer neighborhoods using for instance the 2-flip move as described in Glover and Hao (2009b) would be worth examining. Joining such approaches with associated strategies to focus only on a selected subset of neighbors would enhance their effectiveness, given the computational expense of examining all neighbors at each iteration. Similarly, instead of using the objective function as the unique evaluation measure, other evaluation functions using additional information would likewise be worth exploring. Finally, more advanced adaptive memory strategies from tabu search afford opportunities for creating further improvements.

\section{Acknowledgment}

We are grateful for comments by the referees that have improved the paper. The work is partially supported by a "Chaire d'excellence" from "Pays de la Loire" Region (France) and regional MILES (2007-2009) and RaDaPop projects (2009-2012).

\section{References}

Alidaee B, Kochenberger GA, Ahmadian A (1994) 0-1 quadratic programming approach for the optimal solution of two scheduling problems. International 
Journal of Systems Science 25:401-408

Alidaee B, Kochenberger GA, Lewis K, Lewis M, Wang H (2008) A new approach for modeling and solving set packing problems. European Journal of Operational Research 86(2):504-512

Alkhamis TM, Hasan M, Ahmed MA (1998) Simulated annealing for the unconstrained binary quadratic pseudo-boolean function. European Journal of Operational Research 108:641-652

Amini M, Alidaee B, Kochenberger GA (1999) A scatter search approach to unconstrained quadratic binary programs, McGraw-Hill, New York, NY, pp 317-330. New Methods in Optimization

Beasley JE (1996) Obtaining test problems via internet. Journal of Global Optimization 8:429-433

Beasley JE (1998) Heuristic algorithms for the unconstrained binary quadratic programming problem. Working Paper, The Management School, Imperial College, London, England

Borgulya I (2005) An evolutionary algorithm for the binary quadratic problems. Advances in Soft Computing 2:3-16

Chardaire P, Sutter A (1994) A decomposition method for quadratic zero-one programming. Management Science 41(4):704-712

Gallo G, Hammer P, Simeone B (1980) Quadratic knapsack problems. Mathematical Programming 12:132-149

Glover F, Hao JK (2009a) Efficient evaluations for solving large 0-1 unconstrained quadratic optimization problems. To appear in International Journal of Metaheuristics $1(1)$

Glover F, Hao JK (2009b) Fast 2-flip move evaluations for binary unconstrained quadratic optimization problems. To appear in International Journal of Metaheuristics

Glover F, Laguna M (1997) Tabu Search. Kluwer Academic Publishers, Boston

Glover F, Kochenberger GA, Alidaee B (1998) Adaptive memory tabu search for binary quadratic programs. Management Science 44:336-345

Harary F (1953) On the notion of balanced of a signed graph. Michigan Mathematical Journal 2:143-146

Katayama K, Narihisa H (2001) Performance of simulated annealing-based heuristic for the unconstrained binary quadratic programming problem. European Journal of Operational Research 134:103-119

Katayama K, Tani M, Narihisa H (2000) Solving large binary quadratic programming problems by an effective genetic local search algorithm. In: Proceedings of the Genetic and Evolutionary Computation Conference (GECCO'00), Morgan Kaufmann, pp 643-650

Kochenberger GA, Glover F, Alidaee B, Rego C (2004) A unified modeling and solution framework for combinatorial optimization problems. OR Spectrum 26:237-250

Kochenberger GA, Glover F, Alidaee B, Rego C (2005) An unconstrained quadratic binary programming approach to the vertex coloring problem. Annals of Operations Research 139:229-241 
Krarup J, Pruzan A (1978) Computer aided layout design. Mathematical Programming Study 9:75-94

Laughunn DJ (1970) Quadratic binary programming. Operations Research 14:454-461

Lewis M, Kochenberger GA, Alidaee B (2008) A new modeling and solution approach for the set-partitioning problem. Computers and Operations Research 35(3):807-813

Lodi A, Allemand K, Liebling TM (1999) An evolutionary heuristic for quadratic 0-1 programming. European Journal of Operational Research 119(3):662-670

Lü Z, Hao JK (2009) A critical element-guided perturbation strategy for iterated local search. In: Cotta C, Cowling P (eds) Ninth European Conference on Evolutionary Computation in Combinatorial Optimization (EvoCop 2009), Springer-Verlag, LNCS 5482, pp 1-12

McBride RD, Yormark JS (1980) An implicit enumeration algorithm for quadratic integer programming. Management Science 26:282-296

Merz P, Freisleben B (1999) Genetic algorithms for binary quadratic programming. In: Proceedings of the Genetic and Evolutionary Computation Conference (GECCO'99), Morgan Kaufmann, pp 417-424

Merz P, Freisleben B (2002) Greedy and local search heuristics for unconstrained binary quadratic programming. Journal of Heuristics 8:197-213

Merz P, Katayama K (2004) Memetic algorithms for the unconstrained binary quadratic programming problem. BioSystems 78:99-118

Palubeckis G (2004) Multistart tabu search strategies for the unconstrained binary quadratic optimization problem. Annals of Operations Research 131:259-282

Palubeckis G (2006) Iterated tabu search for the unconstrained binary quadratic optimization problem. Informatica 17(2):279-296

Pardalos P, Rodgers GP (1990) Computational aspects of a branch and bound algorithm for quadratic zero-one programming. Computing 45:131-144

Pardalos P, Xue J (1994) The maximum clique problem. Journal of Global Optimization 4:301-328

Phillips AT, Rosen JB (1994) A quadratic assignment formulation of the molecular conformation problem. Journal of Global Optimization 4:229-241

Witsgall C (1975) Mathematical methods of site selection for electronic system (ems). NBS Internal Report 